\title{
Test Data Generation with a Kalman Filter-Based Adaptive Genetic Algorithm
}

\author{
Aldeida Aleti ${ }^{\mathrm{a}}$, Lars Grunske ${ }^{\mathrm{b}}$ \\ ${ }^{a}$ Faculty of Information Technology, Monash University \\ email: aldeida.aleti@monash.edu \\ ${ }^{b}$ Institute of Software Technology, University of Stuttgart \\ email: lars.grunske@informatik.uni-stuttgart.de
}

\begin{abstract}
Software testing is a crucial part of software development. It enables quality assurance, such as correctness, completeness and high reliability of the software systems. Current state-of-the-art software testing techniques employ search-based optimisation methods, such as genetic algorithms to handle the difficult and laborious task of test data generation. Despite their general applicability, genetic algorithms have to be parameterised in order to produce results of high quality. Different parameter values may be optimal for different problems and even different problem instances. In this work, we introduce a new approach for generating test data, based on adaptive optimisation. The adaptive optimisation framework uses feedback from the optimisation process to adjust parameter values of a genetic algorithm during the search. Our approach is compared to a state of the art test data optimisation algorithm that does not adapt parameter values online, and a representative adaptive optimisation algorithm, outperforming both methods in a wide range of problems.
\end{abstract}

Keywords: test data generation, optimisation, genetic algorithm, adaptive parameter control

\section{Introduction}

Search-based optimisation methods, such as genetic algorithms, ant colony optimisation and simulated annealing have successfully been applied in solving a wide range of software testing problems [41, 30, 2, 31, 40, 21]. The commonality of these approaches is that they aim at optimising the quality of a given test data set. Quality could be measured with respect to certain coverage criteria, eg. branch, statement, and method coverage for source code, or metrics such as transition coverage for model-based testing. Beside these function-oriented approaches, a variety of methods focus on non-functional aspects of test data optimisation, such as searching for input situations that break memory or storage requirements, automatic detection of memory leaks, stress testing and security testing. A systematic literature review of search-based testing for non-functional system properties by Afzal et al. [1] presents a comprehensive overview of these methods.

Genetic algorithms (GAs) are among the most frequently applied search-based optimisation method in test data generation [4. GAs provide approximate results where exact approaches cannot be devised and optimal solutions are hard to find. They can 
be used by practitioners as a 'black box', without mastering advanced theory, whereas more sophisticated exact approaches are tailored to the specific mathematical structure of the problem at hand, and can become completely inapplicable if small aspects of the problem change. Another advantage of GAs compared to traditional methods, such as gradient descent-based algorithms is the stochastic element which helps them get out of local optima.

In recent years, it has been acknowledged that the performance of GAs depend on the numerous parameters, such as crossover rate, mutation rate and population size [19, 17, 3]. Algorithm parameters determine how the search space is explored. They are responsible for the flexibility and efficiency of the search procedure, and when configured appropriately, produce high-quality results regardless of the search space difficulty. Because of the influence of parameter values on algorithm performance, poor algorithm parameterisation hinders the discovery of solutions with high quality [39, 19, 17]. It has been acknowledged that parameters required for optimal algorithm performance are not only domain-specific, but also have to be chosen based on the problem instance at hand [18].

The configuration of the algorithm is usually the responsibility of the practitioner, who often is not an expert in search-based algorithms. General guidelines for successful parameter values give different recommendations: e.g. De Jong [14] recommends using 0.6 for crossover rate, Grefenstette [27] suggests 0.95, whereas Schaffer et al. [48] recommends any value in the range $[0.75,0.95]$. These general guidelines conflict with each other, because each one reports the best parameter configuration of a GA for a particular class of problems. For a newly arising problem, the search space is typically unknown, hence it is hard to formulate any general principles about algorithm parameter configurations for universal applicability [42. In these circumstances, algorithm parameterisation presents itself as an optimisation problem in its own right [19]. To save time, practitioners tend to configure the GA based on a few preliminary runs, where different parameter values are tried in an attempt to fine-tune the algorithm to a particular problem [19]. Depending on the number of parameters and their possible values, investigative trials for parameter optimisations can themselves be attempts to solve a combinatorially complex problem. Even if parameters are tuned to their optimal values, there is no guarantee that the performance of the algorithm will be optimal throughout the optimisation process. In fact, it has been empirically and theoretically demonstrated that different parameter settings are required for different stages of the optimisation process [9, 8, 52, 50, 32, 11, 55]. This means that tuning parameter values before the optimisation process does not guarantee optimal GA performance. This problem has been tackled by many researchers in the optimisation community, who propose to set GA parameter values during the run using feedback from the search, known as adaptive parameter control [17, 25, 22, 12, 13, 33, 34, 36, 38, 49, 56. The intuitive motivation comes from the way the optimisation process unfolds from a more diffused global search, requiring parameter values responsible for the exploration of unseen areas of the search space, to a more focused local optimisation process, requiring parameter values which help with the convergence of the algorithm.

In essence, adaptive parameter control methods monitor the behaviour of a GA run, and use the performance of the algorithm to adapt parameter values, such that successful parameter values are propagated to the next generation. The majority of adaptive parameter control methods found in the literature belong to the class of probability matching 
techniques [12, 13, 33, 34, 36], in which the probability of applying a parameter value is proportional to the quality of that parameter value. The earliest approaches [34, 33] lacked the exploration of parameter values if the feedback from the algorithm was not in their favour in the initial phases of the process. In later work, a minimum selection probability was introduced [34], to ensure that under-performing parameter values did not disappear during the optimisation, in case they were beneficial in the later stages of the search. One of the more recent and mature examples of probability matching is the work by Igel and Kreutz [34, where the selection probability for each parameter value incorporates a minimum probability of selection.

These adaptive parameter control methods assume that the improvement in the quality of the solutions is directly related to the use of certain parameter values. However, GAs are stochastic systems that may produce different results for the same parameter values [16]. Ideally, the randomness induced by the stochastic behaviour of GAs should be taken care of by the parameter control strategy. To address this issue, we introduce an adaptive genetic algorithm, which adjusts algorithm parameters during the optimisation process using a Kalman filter. The Kalman filter-based genetic algorithm (KFGA) redefines parameter values repeatedly based on a learning process that receives its feedback from the optimisation algorithm. The Kalman filter is employed to reduce the noise factor from the stochastic behaviour of GAs when identifying the effect of parameter values on the performance of the algorithm. KFGA outperforms a GA with pre-tuned parameter values and the probability matching technique by Igel and Kreutz [34] when solving the problem of test data generation. The proposed methods consistently finds test suites with better branch coverage than the state-of-the-art, as shown by the experiments performed on a set of open-source problem instances.

\section{Test Data Generation}

Software testing is used to estimate the quality of a software system. Quality may refer to functional attributes, which describe how the system should behave, or nonfunctional requirements as defined in 'IEEE International Standard 14712000 - Systems and software engineering - Recommended practice for architectural description of software-intensive systems' [35], such as reliability, efficiency, portability, maintainability, compatibility and usability.

A software test consists of two main components, an input to the executable program and a definition of the expected outcome. In software testing, the aim is to find the smallest number of test cases that expose as many scenarios as possible in which the system may fail. It is important that the generated test inputs cover as many lines of code as possible. However, since it is usually the task of a human tester to specify the expected outcome, it is also essential to keep the number of test cases as small as possible to make this task feasible.

Due to the time-consuming nature and computational load of this task, optimisation techniques, such as genetic algorithms have been used to automate the process of test case generation [23, 58, 51, 54, 26]. A common approach is to use coverage criteria, such as branch coverage [23] to measure the quality of generated test cases and guide the optimisation process towards the optimal solution(s) that maximise or minimise the criteria. Coverage criteria are a set of coverage goals, which are usually optimised separately [29]. 
However, it is often the case that testing goals conflict with each other, hence should be optimised simultaneously. EvoSuite [23] addresses the problem of conflicting test goals by evolving test cases in a test suite simultaneously with a genetic algorithm, known as whole test suite generation. We use the EvoSuite framework 1 to model the optimisation problem of test suite generation, and extend it to incorporate adaptive optimisation which adjusts the parameter values of the GA during the optimisation process.

\section{Optimisation Model for Test Data Generation}

GAs evolve a population of solutions using the genetic operators: mutation, crossover, selection, and replacement procedure. The aim is to optimise some quality function(s) by searching the space of potential solutions. GAs start the optimisation process by randomly generating a set of solutions. In certain cases, this initial set of solutions is created by applying heuristic rules (e.g. greedy algorithm), or obtained using expert knowledge. Usually, the genetic operators are applied based on predefined rates, such as for crossover or mutation. These operators are employed to create the new solutions by changing excising solutions that are selected according to the selection procedure. The new candidate solutions are added to the pool of population. Finally, the set of solutions that will survive to the next generation are selected using the replacement procedure, which removes as many individuals as required to maintain the prescribed population size.

For test data generation we employ EvoSuite [23], which evolves test suites using a GA with the aim of covering all test goals while minimising the total size of the suite. To measure the quality of a solution, we use branch coverage as an objective function. The aim is to cover as many control structures, such as if and while statements as possible, by evaluating the logical predicates that result in both true and false. In order for all branches to be covered, each predicate should be executed at least twice, resulting in true and false values. In EvoSuite, the branch distance $d(b, T)$ for each branch $b$ in test suite $T$ is defined as

$$
d(b, T)= \begin{cases}0 & \text { if the branch has been covered, } \\ d_{\min }(b, T) & \text { if the predicate has been executed at least twice } \\ 1 & \text { otherwise. }\end{cases}
$$

The distance $d_{\min }(b, T)$ is 0 if at least one of the branches has been covered, and $>0$ otherwise. The fitness function that is minimised by EvoSuite and used in the experiments in Section 6 is formally defined as

$$
f(T)=|M|-\left|M_{T}\right|+\sum_{b \in B} d(b, T),
$$

where $|M|$ is the total number of methods, $\left|M_{T}\right|$ is the number of executed methods in test suite $T$ and $B$ is the set of branches in the program. The optimisation process in EvoSuite starts with a set of solutions, which are uniformly, randomly generated. Formally, let $t$

\footnotetext{
${ }^{1}$ EvoSuite can be downloaded from http://www.evosuite.org.
} 
denote a test case, composed of a sequence of statements $t=\left\langle s_{1}, s_{2}, \ldots, s_{l}\right\rangle$ of length $l$. A statement $s_{i}$ can be a constructor, a field, a primitive, a method or an assignment. A solution is defined as a collection of $T=\left\{t_{1}, t_{2}, \ldots, t_{n}\right\}$ of test cases. The test suite should not only cover as many branches as possible, but also be the shortest possible. Hence, an optimal solution $T^{*}$ is a test suite that covers all possible branches, but at the same time has the smallest possible number of statements. The sum of the lengths of all test cases in a test suite defines its length, i.e.

$$
|T|=\sum_{i=1}^{n}\left|t_{i}\right| .
$$

Since the number of test cases in a test suite and the number of statements in a test case may vary, the solution representation is of a variable size. The solutions are evolved in iterations until a stopping criterion is achieved. The new generations of solutions are created with the help of four genetic operators: the crossover operator, the mutation operator, the selection operator and the replacement operator. The crossover operator creates two new solutions $T_{1}^{\prime}$ and $T_{2}^{\prime}$ by combining test cases from two pre-existing test suites $T_{1}$ and $T_{2}$. A parameter $\alpha$, which is randomly selected in the range $[0,1]$ indicates how the solutions are combined: the first new solution $T_{1}^{\prime}$ receives $\left\lceil\alpha\left|T_{1}\right|\right\rceil$ test cases from $T_{1}$ and the rest from $T_{2}$, whereas the new second solution is composed of the first $\alpha\left|T_{2}\right|$ test cases from the second parent solutions and $\left\lfloor(1-\alpha)\left|T_{1}\right|\right\rfloor$ test cases from the second parent.

The mutation operator is applied after the crossover operator. There are two different types of mutations that can occur: at a test suite level and at a test case level. Test suites are mutated by changing each of the test cases with a probability $1 / n$, where $n$ is the number of test cases in the test suite. At the same time, new test cases are added to the test suite with a probability $\sigma$. Mutation of test cases is performed by either adding, changing or removing statements from a test case with a probability $1 / l$, where $l$ is the number of statements in a test case.

A rank-based selection procedure is employed to select the parent solutions that will undergo recombination and mutation procedures [57]. Solutions are ranked based on the fitness function defined in eq. 2. When there is a tie between solutions, shorter test suites are assigned better ranks. As a result, solutions with better branch coverage and shorter length have a higher chance of projecting their 'genes' to the next generation. Similar to the original studies with EvoSuite, an elitist strategy is used as a replacement procedure [23]. The elitist strategy selects the best solutions to create the next generation.

\section{Adjusting Parameters of Genetic Algorithms}

Genetic algorithms (GAs) have successfully been applied to Whole Test Suite Generation [23] and many other optimisation problems in Software Engineering [46, 28, 5]. The performance of GAs is affected by the configuration of its parameter values, such as crossover rate, mutation rate and population size [10, 20, 15, 6]. Parameter values can be configured in two different ways: by tuning their values before the optimisation process, or by dynamically adjusting parameter assignments during the run. The later is referred to as parameter control, and is more effective than parameter tuning, since different algo- 
rithm configurations may be optimal at different stages of the search [10, 20, 15, 6]. An investigation on the benefit of tuning parameter values for solving software engineering problems showed that tuned parameter values do not necessarily produce better algorithm performance compared to default parameter values [7].

Parameter control methods can be classified into three groups: deterministic, selfadaptive and adaptive. Deterministic parameter control changes parameter values based on a predefined schedule composed of intervals with preassigned parameter values, e.g. decreasing mutation rate by a certain amount every iteration. Using a predefined schedule is likely to lead to suboptimal values for some problems or instances, since smaller problems may require shorter intervals for the schedule, as the search progress will be faster when the problem complexity is lower.

Alternatively, the search for optimal parameters can be integrated into the optimisation process itself - usually by encoding parameter settings into the genotype of the solution to evolve [10, 20, 15], known as self-adaptive parameter control. Extending the solution size to include the parameter space obviously increases the search space and makes the search process more time-consuming [17]. The approach proposed in this work falls into the category of adaptive parameter control, in which feedback from the optimisation process is collected and used to evaluate the effect of parameter value choices and adjust the parameter values over the iterations.

Adaptive parameter control does not use a predefined schedule and does not extend the solution size, which makes it a more effective way for adjusting parameter values. Furthermore, unlike self-adaptive methods, adaptive strategies use feedback from the search to adjust parameter values based on their ability to create high-quality solutions. The genetic operators, and other elements of a GA that are involved in the optimisation process, such as the probabilities of applying the genetic operators, the size of the population, and the number of new solutions produced at every iteration are the parameters of the algorithm that can be adapted by the parameter control method.

Every iteration, the solutions created are evaluated using the objective function, which, in our case, is the branch coverage. The output from the evaluation process is used to assess the effect of the parameter values on the performance of the optimisation algorithm. The approximated effect is employed for a projection of parameter values performing well to the next iterations, based on certain rules, such as the average effect over a number of iterations, instantaneous effect, or the total usage frequency of parameter values. The quality values assigned to the algorithm parameters are used to select the next parameter values, which are employed to create the next generation of solution(s). The selection mechanism configures the components of a GA (variation, selection and replacement operators). The main challenge faced at this step is the trade-off that has to be made between the choice of current best configuration(s) and the search for new optimal settings.

\section{Effect Assessment of Parameter Values using the Kalman Filter}

Formally, given a set $\left\{v_{1}, \ldots, v_{n}\right\}$ of $n$ algorithm parameters, where each parameter $v_{i}$ has $\left\{v_{i 1}, \ldots, v_{i m}\right\}$ values that can be discrete numbers or intervals of continuous numbers, parameter control derives the optimal next value $v_{i j}$ to optimise the effect of $v_{i}$ on the 
performance of the algorithm [3]. As an example, when the mutation rate $v_{1}$ is dynamically adjusted by considering 4 intervals $(m=4), v_{12}$ stands for a mutation rate sampled from the second interval. In the discrete case of optimising the type of mutation operator $v_{2}, v_{22}$ could represent the single-point mutation operator.

Adaptive parameter control methods keep a vector of probabilities denoted as $P=$ $\left\{p\left(v_{11}\right), p\left(v_{12}\right), \ldots, p\left(v_{1 m_{1}}\right), \ldots, p\left(v_{n m_{n}}\right)\right\}$, which represents the selection probability for each parameter value $v_{i j}$. At each time step, the $j^{\text {th }}$ value of the $i^{\text {th }}$ parameter is selected with probability $p\left(v_{i j}\right)$. Its effect on the performance of the algorithm is denoted as $e\left(v_{i j}\right)$. The main goal of adaptive parameter control is to adapt the vector of probabilities $P$ to maximize the expected value of the cumulative effect $E[\mathcal{E}]=\sum_{i=1}^{n} e\left(v_{i j}\right)[3$. The adaptive parameter control employed in this work adjusts parameter values as the optimisation process progresses, using feedback from the search space. Due to the stochastic nature of GAs, the discovery of high-quality solutions may not always be related to algorithm parameters. Instead, the performance of the algorithm is highly susceptible to noise and error, involving random effects of parameter values.

To reduce the noise factor from the stochastic behaviour of GAs, we employ the Kalman filter [37]. The Kalman filter uses a series of measurements observed over time that usually contain noise, and produces a statistically optimal estimate of unknown variables. The algorithm has two main phases: first, it uses previous measurements to predict the current behaviour of parameter values, along with their uncertainties and taking into account noise. In the second phase, once the outcome of the next measurement is observed, which usually contains noise, the estimates are updated using a weighted average, with highly certain estimates being given more weight. As a result, Kalman filter produces reliable estimates of the true performance of parameter values.

\subsection{Kalman Filter Model}

The effect of a parameter value on the performance of the optimisation algorithm is approximated as the fitness change of the solution created by that parameter value compared to the parent solution. More specifically, if a parameter value $v_{i j}$ creates a solution $s_{k}^{v_{i j}}$ by modifying a parent solution $s_{k}$, the effect of $v_{i j}$ on the performance of the optimisation algorithm is calculated as:

$$
e\left(v_{i j}\right)=f\left(s_{k}^{v_{i j}}\right)-f\left(s_{k}\right),
$$

where $f\left(s_{k}^{v_{i j}}\right)$ and $f\left(s_{k}\right)$ are the fitnesses of the child and parent solutions respectively. The observed effect $e\left(v_{i j}\right)$ is stochastic, susceptible to noise and error. This is due to the probabilistic behaviour of GAs, which produce different results for the same parameter values. Hence, instead of using the fitness change to describe the effect of parameter values (eq. 4), the parameter control strategy investigated in this work performs a stochastic estimation of the effect parameter values. To this end, we employ the Kalman filter [37].

Kalman filter has successfully been used for tracking and data prediction problems. It is implemented as a predictor-corrector estimator, which minimises the estimated error covariance. The relationship between the parameter value effect at time $t$ and the effect at the previous iteration $t-1$ is represented by an $n \times n$ matrix $A$. In this paper, we assume $A$ is constant over time. The optional control input $u \in \mathcal{R}^{l}$ is denoted using the $n \times 1$ matrix $B$. Finally, an $m \times n$ matrix $H$ relates the state of the system (parameter 
effect) to measurement $e_{t}^{\prime}$. The filter estimates the state $e \in \mathcal{R}^{n}$ of a process (effect of a parameter value) governed by a linear stochastic difference equation modelled as follows:

$$
e_{t}=A e_{t-1}+B u_{t}+w_{t-1},
$$

In our application of the Kalman filter, $B$ is a zero matrix, since we do not have a control input. Hence, eq. 6 can be simplified to

$$
e_{t}=A e_{t-1}+w_{t-1}
$$

with a measured effect $e_{t}^{\prime} \in \mathcal{R}^{m}$ defined as

$$
e_{t}^{\prime}=H e_{t}+v_{t}
$$

The random variables $w_{t-1}$ and $v_{t}$ represent the process and measurement noise, assumed to be normally distributed and independent of each other. If the noise is not normally distributed, the Kalman filter tries to converge to correct estimations.

The Kalman filter model has previously been used to deal with aspects of uncertainty in GAs. Stroud [53] used the Kalman formulation for stochastic problems, where the fitness function is affected by nonstationary noisy environments. Other works have employed the Kalman model in the context of particle swarm optimisation, such as the Kalman Swarm algorithm [43], which uses the filter to update the position of particles. In this work, we apply the Kalman model for estimating the effect of parameter values on the performance of the algorithm, which to the best of our knowledge has not been previously investigated.

\subsection{Kalman Filter Algorithm}

The feedback control loop of the Kalman filter has two distinct sets of equations: time update equations used in prediction and measurement update equations, necessary for correction. The time update equations are

$$
\hat{e}_{t}^{-}=A \hat{e}_{t-1}
$$

which projects the state ahead, and

$$
P_{t}^{-}=A P_{t-1} A^{T}+Q,
$$

which projects the error covariance $P$ ahead. Matrices A and B are the same as in eq. 6, whereas $Q$ is the process noise covariance matrix. The update equations are

$$
\begin{aligned}
K_{t} & =P_{t}^{-} H^{T}\left(H P_{t}^{-} H^{T}+R\right)^{-1} \\
\hat{e}_{t} & =\hat{e}_{t}^{-}+K_{t}\left(e_{t}^{\prime}-H \hat{e}_{t}^{-}\right) \\
P_{t} & =\left(I-K_{t} H\right) P_{t}^{-} .
\end{aligned}
$$

Equation 10 calculates the Kalman gain $K_{t}$ at time $t$, where $R$ is the measured error covariance. As $R$ approaches zero, the gain $K$ weights the residual more heavily. Equation 11 computes the a posteriori state $\hat{e}_{t}$ from the measurement $\hat{e}_{t}^{-}$. Finally, equation 12 
estimates the a posteriori covariance matrix $P_{t}$. In the next time step, the a posteriori estimates are used to predict the new a priori estimates. This process is performed recursively.

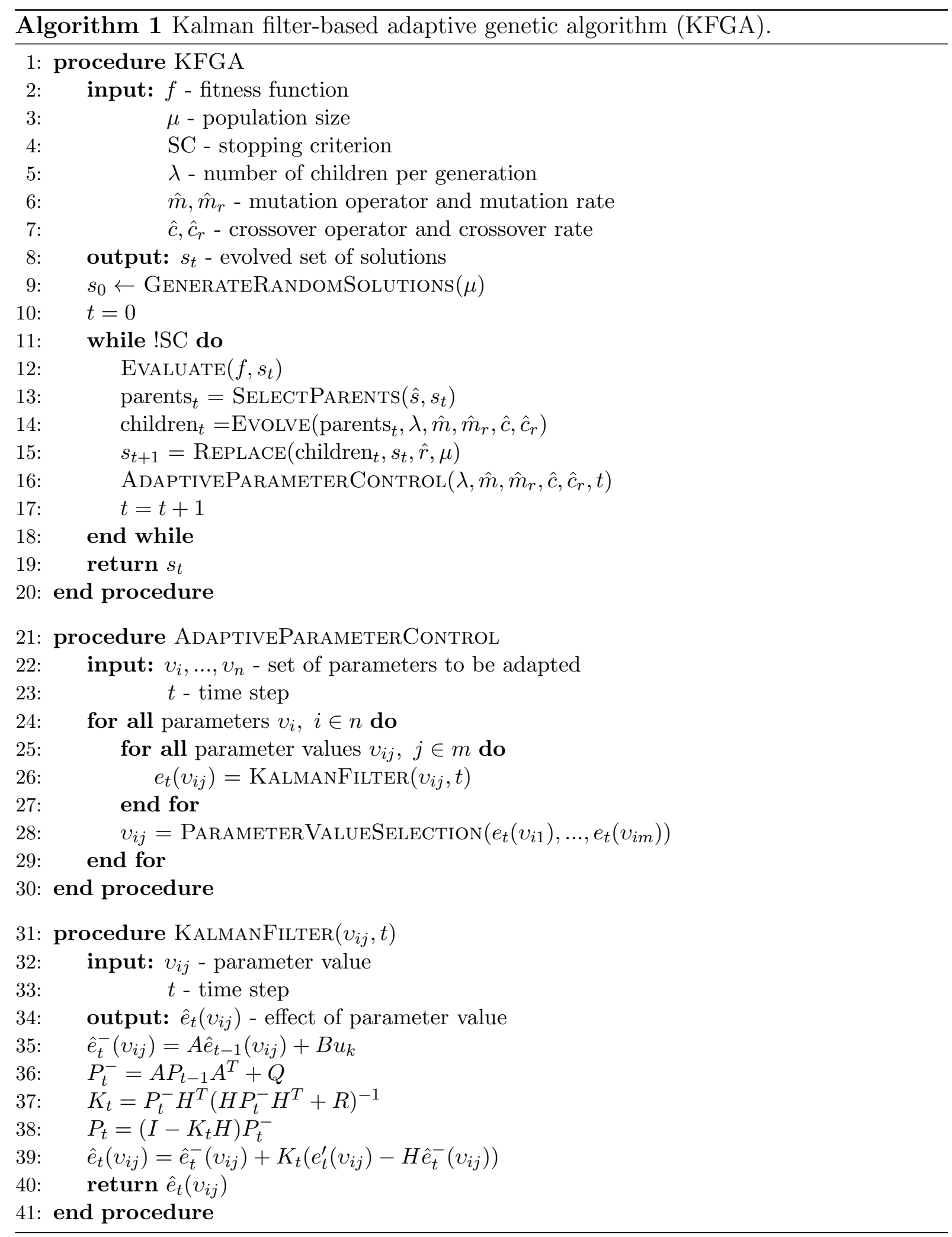


The main steps of the Kalman filter-based adaptive genetic algorithm are described in algorithm 1. The optimisation process starts with a set of solutions $\left(s_{0}\right)$ as initial population, which can be randomly generated, created by applying heuristic rules (e.g. greedy algorithm), or provided by an expert. Line 9 in algorithm 1 describes this process. Parameter $\mu$ is the number of solutions required for each generation. After the initialisation, EA evolves the population using crossover $(\hat{c})$, mutation $(\hat{m})$ and selection $(\hat{s})$ operators until a stopping criterion, defined as ' $\mathrm{SC}$ ' in line 11 is satisfied. The crossover and mutation operators are applied according to the predefined crossover and mutation rates $\hat{c}_{r}$ and $\hat{m}_{r}$. These operators are used to evolve solutions (line 14) that are selected according to the selection procedure (line 13).

The offspring created by the genetic operators are added to the population of solutions. The number of offspring generated every iteration is denoted as $\lambda$. The replacement procedure $\hat{r}$ selects the solutions that will survive in the next generation and removes as many individuals as required to maintain the prescribed population size $\mu$. Next, the adaptive parameter control procedure is invoked to estimate the effect of parameter values using a Kalman filter. The effect of parameter values is used to select the appropriate values for the next iteration using a fitness proportionate selection strategy (line 28). The selection strategy is described in algorithm 2 .

Adaptive parameters are set for an iteration at a time, sampling the respective values probabilistically from the distribution obtained from the Kalman filter. A fitnessproportionate selection mechanism is applied to choose the next parameter configurations, described in algorithm 2. The predicted selection probabilities for values $[1, m]$ of parameter $v_{i}$ are used to associate a probability of selection with each parameter configuration. Initially, a vector of cumulative sums of selection probabilities is created (lines 5-8 in Listing 2). Then a random number is generated in the range [0, sum] (line 9) and matched to the parameter value whose selection probability falls in the interval of the cumulative sums (lines 10-14).

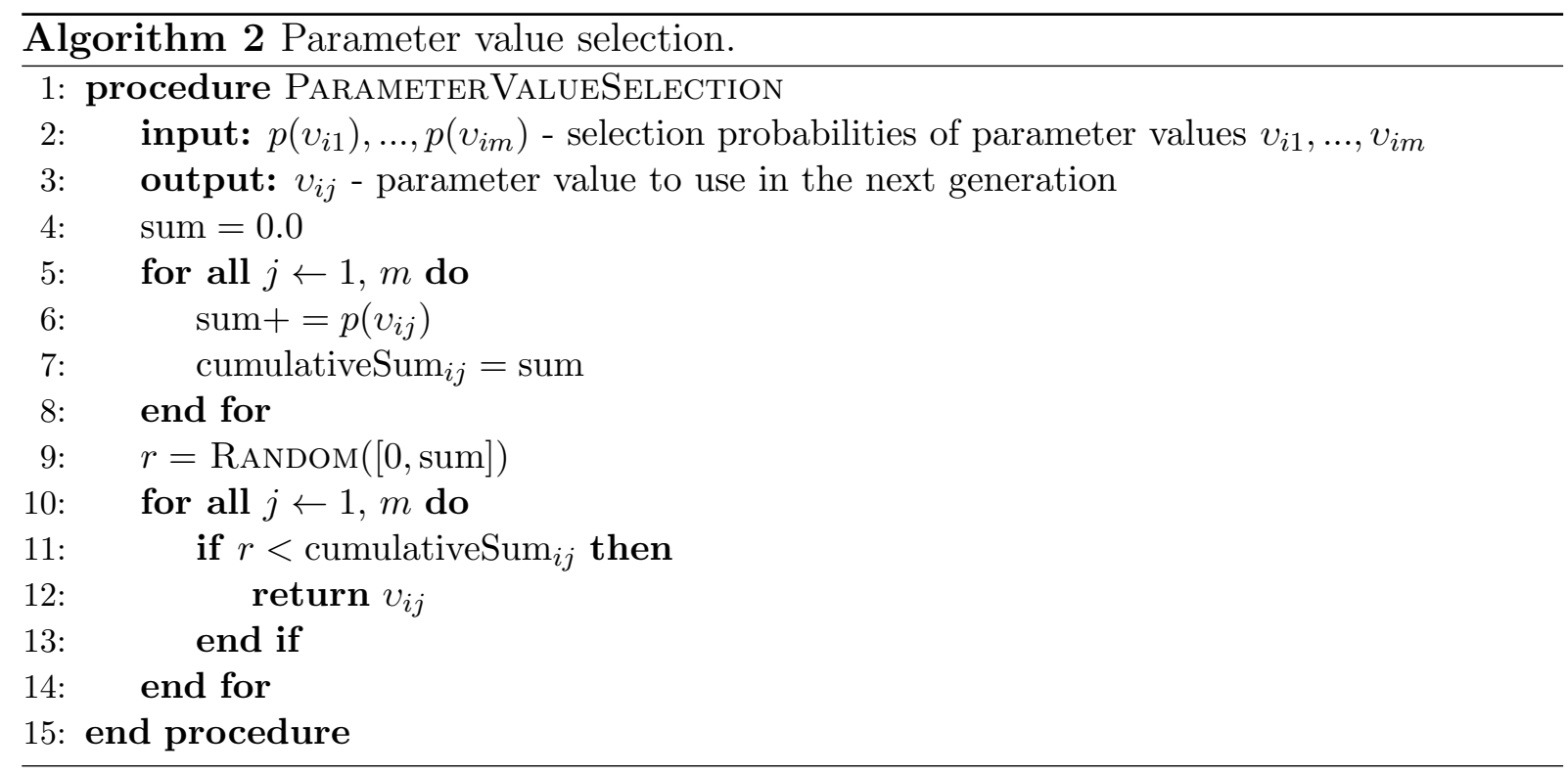

While candidate parameter values with a higher success rate will be less likely to be eliminated, there remains a probability that they may be. With this kind of fitness 
proportionate selection, there is a chance that some weaker solutions may survive the selection process; this is an advantage, as though a parameter value may be not as successful, it may include some component which could prove useful in the following stages of the search.

\section{Experimental Evaluation}

The new adaptive control method is applied to adjust the mutation and crossover rate of a GA during the optimisation process $\mathrm{2}^{2}$. The experiments were conducted using EvoSuite [23], described in Section 3. The Kalman filter-based adaptive parameter control was compared against pre-tuned parameter settings and probability matching technique (PM) [34]. The three optimisation schemes were used to optimise the same set of problem instances. A fixed number of computations was used as a stopping criterion. The problem instances, experimental settings and results are described in the following sections.

\subsection{Problem Instances}

Table 1: The set of open source libraries and programs used to validate the adaptive genetic algorithm. LOC represents the lines of code, $|M|$ is the total number of methods, and $|B|$ is the number of branches.

\begin{tabular}{|c|c|c|c|c|c|c|}
\hline Project & Version & Website & LOC & $|M|$ & $|B|$ & $\frac{|B|}{|M|}$ \\
\hline DAWN & 1.1 .0 & water-simulator.sourceforge.net & 5,433 & 28 & 4 & 0.1 \\
\hline DsaChat & 1.2 .0 & dsachat.sourceforge.net & 2,807 & 950 & 3,224 & 3.4 \\
\hline GreenCow & 2010-04-10 & greencow.sourceforge.net & 4 & 1 & 1 & 1.0 \\
\hline PetSoar & 2013-03-08 & petsoar.sourceforge.net & 3,168 & 101 & 26 & 0.3 \\
\hline TulliBee & 2012-09-23 & sourceforge.net/projects/tullibee & 3,708 & 154 & 840 & 5.5 \\
\hline $\mathrm{A} 4 \mathrm{j}$ & V1.0.1 & a4j.sourceforge.net & 2,826 & 307 & 390 & 1.3 \\
\hline Jwbf & V3.0.0 & jwbf.sourceforge.net & 7,548 & 381 & 572 & 1.5 \\
\hline SaxPath & V1.1.6 & WWW.saxpath.org & 1,919 & 150 & 578 & 3.9 \\
\hline Follow & 1.7 .4 & follow.sourceforge.net & 3,394 & 522 & 560 & 1.4 \\
\hline Dve & V0.3.3 & dvd-homevideo.sourceforge.net & 2,289 & 78 & 114 & 1.5 \\
\hline Res & 2010 & resources4j.sourceforge.net & 938 & 14 & 154 & 11.0 \\
\hline DieBierse & $2009-12-27$ & diebierse.sourceforge.net & 1,647 & 116 & 160 & 1.4 \\
\hline Biff & 2005-06-08 & biff.sourceforge.net & 1,753 & 3 & 12 & 0.7 \\
\hline JGaap & 2007-09-05 & jgaap.sourceforge.net & 4,021 & 54 & 98 & 1.8 \\
\hline Heal & 2002-08-02 & heal.sourceforge.net & 17,213 & 2,810 & 4,906 & 1.8 \\
\hline Lili & V0.9.44 & lilith.huxhorn.de & 54,060 & 748 & 2,258 & 30.5 \\
\hline Lotus & 2007-12-07 & lotus.sourceforge.net & 681 & 69 & 62 & 0.9 \\
\hline
\end{tabular}

To analyse the performance of the optimisation schemes, we selected 17 open source libraries and programs depicted in Table 1. To avoid any bias towards selecting problem instances that are in favour of the proposed method, the instances were selected uniformly at random from SourceForge, and can be downloaded from the websites listed in the third column. They vary in the number of methods, statements and branches. The smallest problem is composed of 1 method and 1 branch (GreenCow), whereas the largest problem

${ }^{2}$ The source code of the GA with adaptive parameter control can be downloaded from http://users. monash.edu.au/ aldeidaa/AdaptiveOptimisation. 
has 748 methods and 2258 branches (Lilith). The second column in Table 1 shows the version or latest update of the programs used in the experiments.

Branch coverage is used as an indicator of the performance of the optimisation algorithm. Branch coverage evaluates whether Boolean expressions tested in control structures (if-statement and while-statement) evaluate to both true and false. The number of branches and their ratio to the number of methods in a problem instance affects this metric, and is an indication of problem difficulty. Hence, we have selected a wide range of problems with different number of branches and branch/method ratios, as depicted in Table 1 .

\subsection{Experimental Settings}

The crossover and mutation operators and their rates are probably the most prominent control parameters to optimise in genetic algorithms [9]. Hence, for the benefit of the experiments, the crossover and mutation rates were varied, with different value intervals to sample from. Preliminary trials have shown that a cardinality of four intervals in the range $\{[0.7,0.775),[0.775,0.85),[0.85,0.925),[0.925,1.0)\}$ produced the best results among several cardinalities with even spreads between 0.7 and 1 for crossover rate, and four intervals in the range [0.001,0.3] for mutation rate.

The parameter values for the pre-tuned GA are based on recommendation from Fraser and Arcuri [23], many of which are 'best practice'. The crossover rate is set to 0.75 , the rate of inserting a new test case is 0.1 , whereas the rate for inserting a new statement is 0.5 .

\subsection{Benchmark Methods}

The proposed method is compared against the probability matching technique (PM), which is one of the most prominent techniques for adjusting GA parameters [34]. PM calculates the selection probability for each parameter value at time step $t$ as

$$
p_{t}\left(v_{i j}\right)= \begin{cases}w /\left(\sum_{r=1}^{m} q_{t}\left(v_{i r}\right)\right) \sum_{r=t-\tau}^{t-1} q_{r}\left(v_{i j}\right)+(1-w) p_{t-1}\left(v_{i j}\right) & \text { if } \sum_{r=1}^{m} q\left(v_{i s}\right)>0 \\ \frac{w}{m}+(1-w) p_{t-1}\left(v_{i j}\right) & \text { otherwise }\end{cases}
$$

where $m$ is the number of possible values for parameter $v_{i}, \tau$ is the time window considered to approximate the quality of a parameter value, and $w \in(0,1]$ is the weight given to each parameter value.

Igel and Kreutz [34] introduce a minimum selection probability $p_{\min }$ for each parameter value, to ensure that under-performing parameter values do not disappear during the optimisation, since they may be beneficial in the later stages of the search. The selection probability for each parameter value is calculated using eq. 14 .

$$
p_{t}^{\prime}\left(v_{i j}\right)=p_{\min }+\left(1-m p_{\min }\right) \frac{p_{t}\left(v_{i j}\right)}{\sum_{r=1}^{m} p_{t}\left(v_{i r}\right)}
$$

Dividing the selection probability by the sum of selection probabilities of all possible parameter values normalises the values in the interval $[0,1]$. 


\subsection{Comparative Measure}

To obtain a fair comparison, the generally accepted approach is the Mean of Solutions Quality (MSQ), which allows the same number of function evaluations for each trial [47]. Therefore, for the current comparison, all trials were repeated 30 times for each optimisation scheme, for 1,000,000 function evaluations, i.e. maximum number of statements created. These values were decided after running the algorithm once for every problem and choosing the value where the quality of the solutions seemed to not improve any further. Nevertheless, there are indications that all algorithms still make small but steady improvements after these numbers of evaluations. MSQ considers the stochastic nature of GAs, which leads to different results for different runs, by expressing the performance measure as the mean of the final quality of the solutions over a number of independent runs. Altogether 30 independent runs are performed per optimisation scheme and test problem in order to restrict the influence of random effects. A different initial population is randomly created each time, and for each test problem all GAs operate on the same 30 initial populations. The MSQ can always be used to measure the performance of stochastic optimisers, since it does not require the optimal solutions. In order to check for a statistical difference in the outperformance of the optimisation schemes, results are validated using the Kolmogorov-Smirnov (KS) non-parametric test [45].

\subsection{Analysis of Results}

The results of the experiments are shown in Table 2, 3 and 4 . For each problem instance and optimisation scheme, we have recorded the test coverage as the percentage of total goals covered, branch coverage as the percentage of branches covered, and the test suite length. Total goals represent method and branch coverage. The most important indicator of the performance of the optimisation algorithm is the branch coverage, since it is the primary objective considered during the optimisation process. In the case of branch and goal coverage, higher mean values indicate better performance, whereas for test suite length lower values are more desirable.

Table 3 shows the mean, standard deviation and KS test values of branch coverage over the 30 runs of the optimisation schemes and problems instances. It can be observed that the Kalman filter-based Genetic algorithm (KFGA) has outperformed the standard genetic algorithm (SGA) and the GA with probability matching parameter control (PM) in the majority of the problem instances with respect to branch coverage. The difference in results is more evident for larger problems, such as Heal (2810 methods, and 4906 branches), TulliBee (154 methods, and 840 branches), and DsaChat (950 methods, and 3224 branches).

In the case of GeenCow and Biff, all optimisation schemes find test suites with $100 \%$ branch coverage. GeenCow is a very small problem, with 1 method and 1 branch. Biff, on the other hand, is a slightly bigger problem, with 3 methods and 2 branches. However, it is still relatively small compared to the other problems considered. A $100 \%$ branch coverage ensures that all branches in terms of control flow are executed at least once. A high branch coverage helps ensure correct functionality. In the larger problem instances, the three optimisation schemes (KFGA, SGA and PM) do not achieve 100\% branch coverage. The larger the problem, the lower the branch coverage is, with DvdHomevideo having the smallest value for branch coverage. Although, this assertion is not always true. For 
Table 2: The mean, standard deviation, and the KS-tests (d-values and p-values) of the 30 runs for branch coverage in the open source libraries and programs when optimised with the Kalman filter-based genetic algorithm (KFGA), the standard genetic algorithm (SGA) and the GA with the probability matching technique $(\mathrm{PM})$.

\begin{tabular}{|l|rrr|rrr|c|c|}
\hline Problem & \multicolumn{4}{|c|}{ Mean } & \multicolumn{3}{|c|}{ Standard deviation } & \multicolumn{2}{c|}{ KS test } \\
\cline { 2 - 9 } instance & SGA & KFGA & PM & SGA & KFGA & PM & KFGA-SGA & KFGA-PM \\
\hline DAWN & $\mathbf{7 6 . 3 \%}$ & $76.3 \%$ & $76.3 \%$ & $0.0 \%$ & $0.0 \%$ & $0.0 \%$ & 1.00 & 1.00 \\
DsaChat & $56.4 \%$ & $\mathbf{6 1 . 9 \%}$ & $57.3 \%$ & $\mathbf{4 . 6 \%}$ & $13.6 \%$ & $3.4 \%$ & 0.03 & 0.00 \\
GreenCow & $100.0 \%$ & $100.0 \%$ & $100.0 \%$ & $0.0 \%$ & $0.0 \%$ & $0.0 \%$ & 1.00 & 1.00 \\
PetSoar & $83.4 \%$ & $\mathbf{8 8 . 5 \%}$ & $87.7 \%$ & $3.7 \%$ & $\mathbf{0 . 9 \%}$ & $0.6 \%$ & 0.00 & 0.03 \\
TulliBee & $74.5 \%$ & $\mathbf{8 3 . 9 \%}$ & $82.8 \%$ & $2.3 \%$ & $\mathbf{1 . 4 \%}$ & $2.8 \%$ & 0.00 & 0.00 \\
A4j & $85.9 \%$ & $\mathbf{9 2 . 3 \%}$ & $88.4 \%$ & $2.6 \%$ & $\mathbf{0 . 8 \%}$ & $2.4 \%$ & 0.00 & 0.01 \\
Jwbf & $67.5 \%$ & $\mathbf{7 5 . 8 \%}$ & $72.0 \%$ & $11.0 \%$ & $\mathbf{0 . 3 \%}$ & $0.4 \%$ & 0.00 & 0.00 \\
SaxPath & $84.4 \%$ & $\mathbf{9 1 . 3 \%}$ & $80.8 \%$ & $2.9 \%$ & $\mathbf{1 . 8 \%}$ & $1.9 \%$ & 0.01 & 0.00 \\
Follow & $42.2 \%$ & $\mathbf{7 4 . 8 \%}$ & $70.0 \%$ & $1.5 \%$ & $\mathbf{1 . 2 \%}$ & $3.6 \%$ & 0.00 & 0.01 \\
DvdHome. & $29.4 \%$ & $\mathbf{3 8 . 3 \%}$ & $33.3 \%$ & $2.1 \%$ & $\mathbf{0 . 9 \%}$ & $3.8 \%$ & 0.00 & 0.00 \\
Resources. & $44.9 \%$ & $\mathbf{5 0 . 5 \%}$ & $45.0 \%$ & $1.8 \%$ & $4.6 \%$ & $\mathbf{0 . 8 \%}$ & 0.00 & 0.00 \\
DieBierse & $24.8 \%$ & $\mathbf{3 4 . 9 \%}$ & $26.1 \%$ & $4.1 \%$ & $\mathbf{0 . 4 \%}$ & $2.6 \%$ & 0.03 & 0.04 \\
Biff & $100.0 \%$ & $100.0 \%$ & $100.0 \%$ & $0.0 \%$ & $0.0 \%$ & $0.0 \%$ & 1.00 & 1.00 \\
JGaap & $78.6 \%$ & $\mathbf{8 4 . 8 \%}$ & $84.4 \%$ & $6.0 \%$ & $3.8 \%$ & $\mathbf{2 . 4 \%}$ & 0.00 & 0.98 \\
Heal & $72.5 \%$ & $\mathbf{8 4 . 3} \%$ & $83.8 \%$ & $4.2 \%$ & $\mathbf{1 . 2 \%}$ & $3.0 \%$ & 0.00 & 0.00 \\
Lilith & $34.7 \%$ & $\mathbf{4 0 . 1 \%}$ & $30.8 \%$ & $\mathbf{2 . 1 \%}$ & $3.1 \%$ & $3.8 \%$ & 0.00 & 0.00 \\
Lotus & $85.8 \%$ & $\mathbf{8 6 . 8 \%}$ & $86.1 \%$ & $\mathbf{0 . 4 \%}$ & $0.9 \%$ & $0.6 \%$ & 0.00 & 0.03 \\
\hline
\end{tabular}

example, in the case of Heal, despite being a problem with a large number of branches, branch coverage is relatively high for all optimisation schemes.

Certain branches are difficult to cover. Uncovered branches could be infeasible, such as private methods not called in any of the public methods, dead code, and abstract methods or methods of abstract classes that are overridden in all concrete subclasses. In such cases, the branch coverage is less than $100 \%$. Infeasible branches may be the cause of infeasible coverage goals, for which no test data can be generated. Examples are DvdHomevideo and DieBierse, which have the lowest branch coverage of all problems.

As our method outperforms the GA with pre-tuned parameter values and the probability matching technique in the majority of the problems, we employ the KolmogorovSmirnov (KS) non-parametric test [45] to check for a statistical difference in the results. The 30 branch coverage values of the repeated trials for each of the problem instances were submitted to the KS analysis. KFGA was compared to the standard genetic algorithm (KFGA-SGA) and the GA with probability matching technique (KFGA-PM), with a null hypothesis of no significant difference in the performance. The results of the tests are shown in Table 2. All KS tests, used for establishing that there is no difference between independent datasets under the assumption that they are not normally distributed, resulted in a rejection of the null hypothesis with a minimum d-value equal to 0.32 at a $95 \%$ confidence level in most cases apart from the smaller problems, where both optimisation 
schemes found the optimal solution. Hence we conclude that the superior performance of KFGA is statistically significant.

Results of the goal coverage (method and branch coverage) for all problem instances and optimisation schemes are shown in Table 3. Similar to branch coverage, we record the means and standard deviations of 30 runs of goal coverage for all methods, and perform a $\mathrm{KS}$ analysis for statistical significance. It can be observed that in general the mean goal coverage of KFGA is higher than the other methods. When solving small problems, such as GreenCow and Biff, all methods find the same-quality solutions. KFGA is outperformed by SGA in PetSoar. However, the KS analysis in this case shows that the difference in the results is not statistically significant, with p equal to 0.11 .

One interesting observation is the absence of test suites with $100 \%$ goal coverage. One reason for this is the infeasibility of test goals [24], which is caused by infeasible test branches or private methods. This affects the performance of the algorithm in such a way that tuning or controlling parameter values does not have any effect on the goal coverage criterion. Examples are the generation of test data for Resources4J, which results in very low goal coverage $(7.0 \%)$, and Lilith, which has a test suite of $14.4 \%$ goal coverage. Dead code may create infeasible branches or unreachable methods, which results in infeasible test goals. In this example, the KS analysis indicates no statistical significance in the difference between the performance of the optimisation schemes.

Table 3: The means, standard deviations, and the KS-tests (d-value and p-value) for goal coverage of the open source libraries and programs when optimised with the adaptive genetic algorithm (KFGA) and the standard genetic algorithm (SGA).

\begin{tabular}{|c|c|c|c|c|c|c|c|c|}
\hline \multirow{2}{*}{$\begin{array}{l}\text { Problem } \\
\text { instance }\end{array}$} & \multicolumn{3}{|c|}{ Mean } & \multicolumn{3}{|c|}{ Standard deviation } & \multicolumn{2}{|c|}{ KS test } \\
\hline & SGA & KFGA & $\mathrm{PM}$ & SGA & KFGA & $\mathrm{PM}$ & KFGA-SGA & KFGA-PM \\
\hline DAWN & $58.9 \%$ & $66.9 \%$ & 43.8 & $0.0 \%$ & $0.0 \%$ & $0.0 \%$ & 1.00 & 1.00 \\
\hline DsaChat & $26.1 \%$ & $31.6 \%$ & $23.8 \%$ & $1.3 \%$ & $15.4 \%$ & $2.3 \%$ & 0.03 & 0.00 \\
\hline GreenCow & $75.0 \%$ & $75.0 \%$ & $75.0 \%$ & $0.0 \%$ & $0.0 \%$ & $0.0 \%$ & 1.00 & 1.00 \\
\hline PetSoar & $28.4 \%$ & $27.1 \%$ & $25.9 \%$ & $4.7 \%$ & $5.1 \%$ & $1.1 \%$ & 0.11 & 0.11 \\
\hline TulliBee & $34.2 \%$ & $37.8 \%$ & $29.6 \%$ & $1.7 \%$ & $1.1 \%$ & $3.3 \%$ & 0.00 & 0.00 \\
\hline $\mathrm{A} 4 \mathrm{j}$ & $46.5 \%$ & $\mathbf{5 9 . 6} \%$ & $42.1 \%$ & $1.4 \%$ & $1.6 \%$ & $33.6 \%$ & 0.00 & 0.05 \\
\hline Jwbf & $25.4 \%$ & $30.1 \%$ & $26.7 \%$ & $2.6 \%$ & $2.6 \%$ & $0.9 \%$ & 0.00 & 0.00 \\
\hline SaxPath & $33.7 \%$ & $70.6 \%$ & $38.9 \%$ & $2.3 \%$ & $2.1 \%$ & $34.6 \%$ & 0.01 & 0.02 \\
\hline Follow & $17.7 \%$ & $36.4 \%$ & 31.45 & $0.5 \%$ & $0.1 \%$ & $3.3 \%$ & 0.00 & 0.04 \\
\hline DvdHome. & $19.4 \%$ & $28.3 \%$ & $24.4 \%$ & $0.3 \%$ & $0.1 \%$ & $3.2 \%$ & 0.00 & 0.01 \\
\hline Resources4J & $6.0 \%$ & $7.0 \%$ & $6 \%$ & $1.8 \%$ & $0.9 \%$ & $1.9 \%$ & 0.00 & 0.68 \\
\hline DieBierse & $24.8 \%$ & $26.2 \%$ & $12.9 \%$ & $0.2 \%$ & $0.1 \%$ & $1.6 \%$ & 0.03 & 0.01 \\
\hline Biff & $77.8 \%$ & $77.8 \%$ & $77.8 \%$ & $0.0 \%$ & $0.0 \%$ & $0.0 \%$ & 1.00 & 1.00 \\
\hline JGaap & $40.8 \%$ & $47.3 \%$ & $39.0 \%$ & $10.8 \%$ & $9.0 \%$ & $2.2 \%$ & 0.11 & 0.03 \\
\hline Heal & $33.2 \%$ & $56.9 \%$ & $31.7 \%$ & $1.2 \%$ & $0.2 \%$ & $2.8 \%$ & 0.00 & 0.00 \\
\hline Lilith & $8.7 \%$ & $14.4 \%$ & $12.9 \%$ & $2.6 \%$ & $1.7 \%$ & $2.8 \%$ & 0.00 & 0.00 \\
\hline Lotus & $37.2 \%$ & $40.8 \%$ & $38.2 \%$ & $5.6 \%$ & $6.8 \%$ & $1.1 \%$ & 0.03 & 0.03 \\
\hline
\end{tabular}


The mean lengths of the test suites produced by the two optimisation schemes are shown in Table 4. DvdHomevideo is a medium size problem instance. For this problem, the adaptive method has found a test suite which is much shorter in length (more than 11 times shorter) than the test suite produced by the standard GA. In addition, the KFGA optimisation scheme has achieved better branch coverage and goal coverage compared to the SGA. The probability matching technique performs better than SGA, but is outperformed by KFGA.

For small problems like DAWN (28 methods and 4 branches), Biff (3 methods and 2 branches), SGA and KFGA exhibit similar performance, producing test suites with very similar length. The test suite produced by PM, however, is very large compared to the other two method. In terms of the number of statements that were executed, which indicates the efficiency of the algorithm, KFGA outperforms the other two optimisation schemes. For Biff, KFGA has produced a shorter test suite (54 vs. 183 test cases), whereas for Jwbf the test suite produced by SGA is shorter (160 vs. 217). These problem instances are easy to solve, hence the parameter values do not affect the performance of the algorithm greatly.

The adaptive method (KFGA) struggles to produce test suites with smaller length than the ones found by SGA in certain problem instances, such as TulliBee (1652 vs. 1813) and Jwbf (160 vs. 217). It is clear that the adaptive algorithm has spent most of the resources in maximising the branch coverage. In our experiments, the maximisation of branch coverage and the minimisation of the test suite length are not performed simultaneously, which makes it a single-objective optimisation problem. We believe that by converting the problem to multi-objective, and optimising branch coverage and test suite length simultaneously, i.e. by considering the Pareto optimal solutions [44, the adaptive optimisation algorithms will produce better results.

Each optimisation scheme was granted 1,000,000 function evaluations to solve each of the problem instances. However, often the optimisation algorithms stopped earlier. This can happen if a test case with $100 \%$ branch coverage is found before all function evaluations are consumed. For an indication of the resources used by each optimisation scheme, we recorded the average number of executed statements over 30 runs by each optimisation scheme. Results are shown in Table 4.

It can clearly be observed that the PM and KFGA converge faster and use fewer function evaluation than SGA. PM is faster than KFGA in some of the problems, however, it fails to find solutions with better branch coverage, goal coverage and test suite length compared to KFGA. The biggest difference in the number of statements executed is observed for problem Biff, where KFGA is approximately 1493 times faster than SGA, and 213 times faster than PM. Biff is one of the problem instances that were solved to optimum by all three optimisation schemes. This means that if a test suite with $100 \%$ branch coverage is feasible, KFGA is capable of finding it in fewer function evaluations that SGA and PM. Furthermore, these results show that the proposed optimisation strategy is efficient. KFGA is capable of adapting the strategy parameters of a GA to the problem being solved, and as a results improving its performance in efficient time.

The rates of selecting the crossover and mutation probabilities over time are illustrated in Figure 1 for one run of the problem instance Heal. A distinctive difference can be observed between these two algorithm parameters. In the initial iterations, higher mutation 
Table 4: The mean test suite length, and the mean number of statements executed for each open source library and program when optimised with the adaptive genetic algorithms (KFGA) and the standard genetic algorithm (SGA).

\begin{tabular}{|l|rrr|rrr|}
\hline Problem & \multicolumn{3}{|c|}{ Length } & \multicolumn{3}{|c|}{ Number of statements } \\
\cline { 2 - 7 } instance & SGA & KFGA & PM & SGA & KFGA & PM \\
\hline DAWN & $\mathbf{1 6 9}$ & 201 & 1,215 & $1,006,450$ & $\mathbf{1 1 6 , 8 1 2}$ & 865,146 \\
DsaChat & 99 & $\mathbf{9 8}$ & 500 & 645,536 & $\mathbf{5 2 , 0 9 8}$ & 113,119 \\
GreenCow & 2 & 2 & 16 & 5,617 & $\mathbf{5 , 0 9 7}$ & 5,630 \\
PetSoar & 569 & $\mathbf{4 0 4}$ & 435 & $10,389,713$ & $\mathbf{6 5 5 , 9 8 6}$ & 464,339 \\
TulliBee & $\mathbf{1 , 6 5 2}$ & 1,813 & 1,849 & $6,551,126$ & $2,155,416$ & $\mathbf{5 6 7 . 3 5 0}$ \\
A4j & 6,941 & $\mathbf{3 , 3 6 7}$ & 7,218 & $21,961,264$ & $4,547,900$ & $\mathbf{2 , 3 5 3 , 9 2 9}$ \\
Jwbf & $\mathbf{1 6 0}$ & 217 & 884 & $3,136,824$ & $1,326,044$ & $\mathbf{6 2 7 , 6 6 6}$ \\
SaxPath & $\mathbf{3 9 2}$ & 1,028 & 1,058 & $6,395,148$ & $1,034,359$ & $\mathbf{3 0 5 , 0 7 1}$ \\
Follow & 7,288 & $\mathbf{2 , 4 8 8}$ & 3,153 & $1,462,369$ & $\mathbf{9 0 1 , 7 4 8}$ & $1,164,283$ \\
DvdHomeVideo & 713 & $\mathbf{6 3}$ & 71 & $2,838,512$ & 312,583 & $\mathbf{7 0 , 6 2 8}$ \\
Resources4J & 254 & $\mathbf{1 6}$ & 62 & $1,323,240$ & 281,310 & $\mathbf{9 3 , 1 3 8}$ \\
DieBierse & 1,399 & $\mathbf{1 0 6}$ & 459 & $1,041,220$ & 467,795 & $\mathbf{1 2 1 , 6 3 4}$ \\
Biff & 183 & $\mathbf{5 4}$ & 67 & 771,994 & $\mathbf{5 1 7}$ & 110,422 \\
JGaap & 799 & $\mathbf{3 3 0}$ & 610 & $7,855,304$ & $1,066,733$ & 342,959 \\
Heal & 20,128 & $\mathbf{1 4 , 0 9 6}$ & 15,160 & $52,695,245$ & $71,317,028$ & $\mathbf{7 , 8 6 4 , 1 2 3}$ \\
Lilith & 14,205 & 10,546 & $\mathbf{8 , 3 3 6}$ & $12,752,073$ & $3,689,768$ & $\mathbf{3 , 0 1 5 , 6 0 5}$ \\
Lotus & $\mathbf{3 , 2 4 6}$ & 3,491 & 24,596 & $7,397,925$ & $\mathbf{6 5 9 , 5 3 5}$ & $2,629,219$ \\
\hline
\end{tabular}

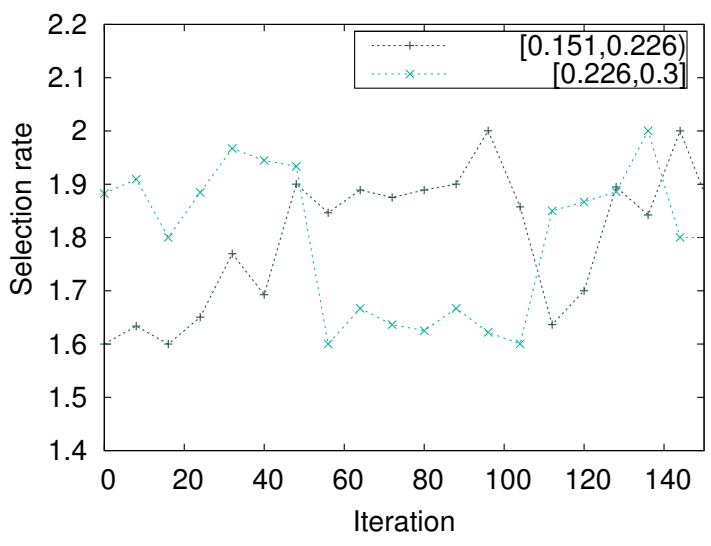

(a) Mutation rate.

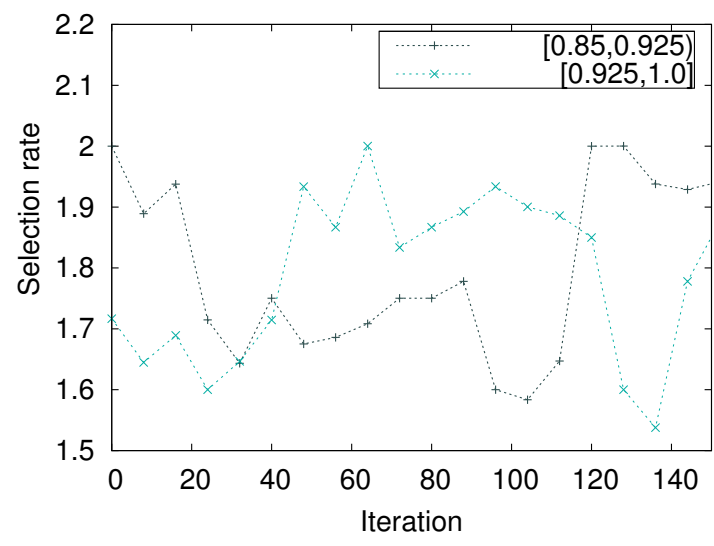

(b) Crossover rate.

Figure 1: The development of selection rates for mutation rates in the ranges $[0.151,0.226)$ and $[0.226,0.3]$, and crossover rate in the ranges $[0.85,0.925)$ and $[0.925,1.0]$.

rates and lower crossover rates are more successful. After a few iterations, KFGA identifies lower mutation rates in the range $[0.151,0.226)$ as performing better. At the same time, the probability of selecting higher crossover rates in the range [0.925,1.0] steadily increases until iteration 115. After this point, lower crossover rates become again more 
successful than lower crossover rates. The selection probabilities of different ranges of mutation rate, on the other hand, become almost equal towards the end of the optimisation process. This means that the mutation operator is not discovering solutions with better quality, despite the changes in the mutation rate. In the future, we will investigate the relationship between the behaviour of different parameter values and the structure of the search space of different problems instances.

\section{Conclusion}

We presented a Kalman filter-based genetic algorithm (KFGA) for solving the problem of test case generation in software testing. The method uses a Kalman filter to reduce the effect of the stochastic behaviour of GAs when estimating the appropriate parameter values to use in each iteration of the optimisation process. KFGA was implemented in EvoSuite, a framework for whole test suite generation. A set of experiments were conducted using open-source libraries and programs with different characteristics, such as number of methods, number of branches and number of testing goals. The performance of the KFGA was compared to the performance of a standard GA with pre-tuned parameter setting and a GA with probability matching parameter control. Results showed that the adaptive method outperformed the standard GA and the probability matching method over the majority of the problem instances.

In some cases, the difference in the performance of the algorithms was not statistically significant. This usually happens either when problems are easy to solve by all optimisation schemes, so controlling parameter values does not have any benefit, or when branches are infeasible, such that no method is able to find any solutions. In general, KFGA not only performed better than the other method, but also used fewer function evaluations, which reflects its efficiency in adapting the search strategy to the problem being solved. In the future, the test suite generation problem will be converted to a multi-objective problem, where branch coverage and the length of the test suite will be modelled as conflicting objectives and optimised simultaneously.

\section{Acknowledgements}

We would like to thank Gordon Fraser and the rest of the team who developed EvoSuite for making the source code available. We wish to acknowledge Monash University for the use of their Nimrod software in this work. The Nimrod project has been funded by the Australian Research Council and a number of Australian Government agencies, and was initially developed by the Distributed Systems Technology. This research was supported under Australian Research Council's Discovery Projects funding scheme (project number DE140100017). We would like to thank several anonymous reviewers who helped improve the quality of this work.

\section{References}

[1] Wasif Afzal, Richard Torkar, and Robert Feldt. A systematic review of search-based testing for non-functional system properties. Information \& Software Technology, 51(6):957-976, 2009. 
[2] Moataz A. Ahmed and Irman Hermadi. GA-based multiple paths test data generator. Computers \& OR, 35(10):3107-3124, 2008.

[3] Aldeida Aleti. An adaptive approach to controlling parameters of evolutionary algorithms. PhD thesis, Swinburne University of Technology, 2012.

[4] Aldeida Aleti, Barbora Buhnova, Lars Grunske, Anne Koziolek, and Indika Meedeniya. Software architecture optimization methods: A systematic literature review. IEEE Transactions on Software Engineering, 39(5):658-683, 2013.

[5] Aldeida Aleti, Lars Grunske, Indika Meedeniya, and Irene Moser. Let the ants deploy your software - an ACO based deployment optimisation strategy. In ASE, pages 505509. IEEE Computer Society, 2009.

[6] Aldeida Aleti and Irene Moser. Entropy-based adaptive range parameter control for evolutionary algorithms. In Proceeding of the fifteenth annual conference on Genetic and evolutionary computation conference, GECCO '13, pages 1501-1508. ACM, 2013.

[7] Andrea Arcuri and Gordon Fraser. Parameter tuning or default values? An empirical investigation in search-based software engineering. Empirical Software Engineering, 18(3):594-623, 2013.

[8] Thomas Bäck. The interaction of mutation rate, selection, and self-adaptation within a genetic algorithm. In Parallel Problem Solving from Nature 2,PPSN-II, pages 8796. Elsevier, 1992.

[9] Thomas Bäck, Ágoston Endre Eiben, and Nikolai A. L. van der Vaart. An empirical study on GAs without parameters. In Parallel Problem Solving from Nature - PPSN VI (6th PPSN'2000), volume 1917 of Lecture Notes in Computer Science (LNCS), pages 315-324. Springer-Verlag (New York), 2000.

[10] Thomas Bäck and Martin Schütz. Intelligent mutation rate control in canonical genetic algorithms. Lecture Notes in Computer Science, 1079:158-167, 1996.

[11] Jorge Cervantes and Christopher R. Stephens. Limitations of existing mutation rate heuristics and how a rank GA overcomes them. IEEE Transcation on Evolutionary Computation, 13(2):369-397, 2009.

[12] David Corne, Martin J. Oates, and Douglas B. Kell. On fitness distributions and expected fitness gain of mutation rates in parallel evolutionary algorithms. In Parallel Problem Solving from Nature - PPSN VII (7th PPSN'02), volume 2439 of Lecture Notes in Computer Science (LNCS), pages 132-141. Springer-Verlag, 2002.

[13] Lawrence Davis. Adapting operator probabilities in genetic algorithms. In Proceedings of the Third International Conference on Genetic Algorithms, pages 70-79. Morgan Kaufman, 1989.

[14] Kenneth A. De Jong. An analysis of the behavior of a class of genetic adaptive systems. PhD thesis, University of Michigan, 1995. 
[15] Kalyanmoy Deb and Hans-Georg Beyer. Self-adaptive genetic algorithms with simulated binary crossover. Evolutionary Computation, 9(2):197-221, 2001.

[16] Kenneth DeJong. Parameter setting in EAs: a 30 year perspective. In Fernando G. Lobo, Cláudio F. Lima, and Zbigniew Michalewicz, editors, Parameter Setting in Evolutionary Algorithms, volume 54 of Studies in Computational Intelligence, pages 1-18. Springer, 2007.

[17] Ágoston Endre Eiben, Robert Hinterding, and Zbigniew Michalewicz. Parameter control in evolutionary algorithms. IEEE Transations on Evolutionary Computation, $3(2): 124-141,2007$.

[18] Ágoston Endre Eiben and Martijn C. Schut. New ways to calibrate evolutionary algorithms. In Patrick Siarry and Zbigniew Michalewicz, editors, Advances in Metaheuristics for Hard Optimization, Natural Computing Series, pages 153-177. Springer, 2008.

[19] Ágoston Endre Eiben and Selmar K. Smit. Parameter tuning for configuring and analyzing evolutionary algorithms. Swarm and Evolutionary Computation, 1(1):1931, 2011.

[20] Raziyeh Farmani and Jonathan A. Wright. Self-adaptive fitness formulation for constrained optimization. IEEE Transaction Evolutionary Computation, 7(5):445-455, 2003.

[21] Javier Ferrer, J. Francisco Chicano, and Enrique Alba. Evolutionary algorithms for the multi-objective test data generation problem. Softw, Pract. Exper, 42(11):13311362, 2012.

[22] Álvaro Fialho, Luis Da Costa, Marc Schoenauer, and Michèle Sebag. Analyzing bandit-based adaptive operator selection mechanisms. Annals of Mathematics and Artificial Intelligence - Special Issue on Learning and Intelligent Optimization, 2010.

[23] Gordon Fraser and Andrea Arcuri. Whole test suite generation. IEEE Transactions on Software Engineering, 39(2):276-291, 2013.

[24] A. Goldberg, T.C. Wang, and D. Zimmerman. Applications of feasible path analysis to program testing. In ACM SIGSOFT International Symposium in Software Testing and Analysis, Proceedings, pages 80-94, 1994.

[25] David E. Goldberg. Genetic Algorithms in Search, Optimization and Machine Learning. Addison-Wesley, 1989.

[26] Dunwei Gong and Yan Zhang. Generating test data for both path coverage and fault detection using genetic algorithms. Frontiers of Computer Science, 7(6):822-837, 2013.

[27] John J. Grefenstette. Optimization of control parameters for genetic algorithms. IEEE Transactions on Systems, Man, and Cybernetics, SMC-16(1):122-128, 1986. 
[28] Lars Grunske. Identifying "good" architectural design alternatives with multiobjective optimization strategies. In International Conference on Software Engineering, ICSE, pages 849-852. ACM, 2006.

[29] Mark Harman, Sung Gon Kim, Kiran Lakhotia, Phil McMinn, and Shin Yoo. Optimizing for the number of tests generated in search based test data generation with an application to the oracle cost problem. In ICST Workshops, pages 182-191. IEEE Computer Society, 2010.

[30] Mark Harman, Kiran Lakhotia, and Phil McMinn. A Multi-Objective Approach To Search-Based Test Data Generation. In Dirk Thierens, editor, 2007 Genetic and Evolutionary Computation Conference (GECCO'2007), volume 1, pages 1098-1105, London, UK, July 2007. ACM Press.

[31] Mark Harman and Phil McMinn. A theoretical and empirical study of search-based testing: Local, global, and hybrid search. IEEE Transaction Software Engineering, 36(2):226-247, 2010.

[32] J. Hesser and R. Manner. Towards an optimal mutation probability for genetic algorithms. Lecture Notes in Computer Science, 496:23-32, 1991.

[33] Tzung-Pei Hong, Hong-Shung Wang, and Wei-Chou Chen. Simultaneously applying multiple mutation operators in genetic algorithms. Journal of Heuristics, 6(4):439455, 2000.

[34] Christian Igel and Martin Kreutz. Operator adaptation in evolutionary computation and its application to structure optimization of neural networks. Neurocomputing, 55(1-2):347-361, 2003.

[35] ISO/IEC. IEEE international standard 14712000 - systems and software engineering - recommended practice for architectural description of software-intensive systems, 2000 .

[36] Bryant A. Julstrom. What have you done for me lately? Adapting operator probabilities in a steady-state genetic algorithm. In Proceedings of the Sixth International Conference on Genetic Algorithms, pages 81-87, San Francisco, CA, 1995. Morgan Kaufmann.

[37] R. E. Kalman. A new approach to linear filtering and predictive problems. Transactions ASME, Journal of basic engineering, (82):34-45, 1960.

[38] F. G. Lobo and David E. Goldberg. Decision making in a hybrid genetic algorithm. In Proceedings of the Congress on Evolutionary Computation, pages 121-125. IEEE Press, 1997.

[39] Fernando G. Lobo. Idealized dynamic population sizing for uniformly scaled problems. In 13th Annual Genetic and Evolutionary Computation Conference, GECCO 2011, Proceedings, pages 917-924. ACM, 2011. 
[40] Stefan Mairhofer, Robert Feldt, and Richard Torkar. Search-based software testing and test data generation for a dynamic programming language. In Natalio Krasnogor and Pier Luca Lanzi, editors, 13th Annual Genetic and Evolutionary Computation Conference, GECCO 2011, Proceedings, Dublin, Ireland, July 12-16, 2011, pages 1859-1866. ACM, 2011.

[41] Phil McMinn. Search-based software test data generation: a survey. Softw. Test, Verif. Reliab, 14(2):105-156, 2004.

[42] Melanie Mitchell. An Introduction to Genetic Algorithms. Complex Adaptive Systems. MIT-Press, Cambridge, 1996.

[43] Christopher K. Monson and Kevin D. Seppi. The kalman swarm: A new approach to particle motion in swarm optimization. In Genetic and Evolutionary Computation, volume 3102 of Lecture Notes in Computer Science, pages 140-150. Springer-Verlag, 2004.

[44] Vilfredo Pareto. CoursD'Economie Politique. F. Rouge, 1896.

[45] A. N. Pettitt and M. A. Stephens. The kolmogorov-smirnov goodness-of-fit statistic with discrete and grouped data. Technometrics, 19(2):205-210, 1977.

[46] Outi Räihä. A survey on search-based software design. Computer Science Review, 4(4):203-249, 2010.

[47] Ronald L. Rardin and Reha Uzsoy. Experimental evaluation of heuristic optimization algorithms:A tutorial. Journal of Heuristics, 7(3):261-304, 2001.

[48] J. David Schaffer, Richard A. Caruana, Larry J. Eshelman, and Rajarshi Das. A study of control parameters affecting online performance of genetic algorithms for function optimization. In Proceedings of the Third International Conference on Genetic Algorithms, pages 51-60. Morgan Kaufman, 1989.

[49] D. Schlierkamp-Voosen and H. Mühlenbein. Strategy adaptation by competing subpopulations. Lecture Notes in Computer Science, 866:199-208, 1994.

[50] Jim Smith and Terence C. Fogarty. Self adaptation of mutation rates in a steady state genetic algorithm. In International Conference on Evolutionary Computation, pages 318-323, 1996.

[51] Praveen Ranjan Srivastava. Optimisation of software testing using genetic algorithm. IJAISC, 1(2/3/4):363-375, 2009.

[52] Christopher R. Stephens, I. Garcia Olmedo, J. Mora Vargas, and Henri Waelbroeck. Self-adaptation in evolving systems. Artificial Life, 4(2):183-201, 1998.

[53] Phillip D. Stroud. Kalman-extended genetic algorithm for search in nonstationary environments with noisy fitness evaluations. IEEE Transactions on Evolutionary Computation, 5(1):66-77, 2001. 
[54] Yeresime Suresh and Santanu Ku. Rath. A genetic algorithm based approach for test data generation in basis path testing. CoRR, abs/1401.5165, 2014.

[55] Dirk Thierens. Adaptive mutation rate control schemes in genetic algorithms. In Proceedings of the 2002 Congress on Evolutionary Computation CEC2002, pages 980-985. IEEE Press, 2002.

[56] Andrew Tuson and Peter Ross. Adapting operator settings in genetic algorithms. Evolutionary Computation, 6(2):161-184, 1998.

[57] Darrell Whitley. The GENITOR algorithm and selection pressure: Why rank-based allocation. In James D. Schaffer, editor, Proc. of the Third Int. Conf. on Genetic Algorithms, pages 116-121, San Mateo, CA, 1989. Morgan Kaufmann.

[58] Andreas Windisch. Search-based test data generation from stateflow statecharts. In Genetic and Evolutionary Computation Conference, GECCO 2010, Proceedings, pages 1349-1356. ACM, 2010. 